Research Article

\title{
Reconstruction and Analysis of Tight Sandstone Digital Rock Combined with X-Ray CT Scanning and Multiple-Point Geostatistics Algorithm
}

\author{
Yun Lei $\mathbb{D i D}^{1,2,3}$ \\ ${ }^{1}$ Shenyang Research Institute, China Coal Technology \& Engineering Group Corp, Fushun 113122, China \\ ${ }^{2}$ State Key Laboratory of Coal Mine Safety Technology, Fushun 113122, China \\ ${ }^{3}$ School of Emergency Management and Safety Engineering, China University of Mining and Technology, Beijing 100083, China
}

Correspondence should be addressed to Yun Lei; yunlei_78@163.com

Received 6 January 2020; Revised 2 April 2020; Accepted 21 April 2020; Published 23 June 2020

Academic Editor: Vassilios Constantoudis

Copyright (c) 2020 Yun Lei. This is an open access article distributed under the Creative Commons Attribution License, which permits unrestricted use, distribution, and reproduction in any medium, provided the original work is properly cited.

Unconventional rocks such as tight sandstone and shale usually develop multiscale complex pore structures, with dimensions ranging from nanometers to millimeters, and the full range can be difficult to characterize for natural samples. In this paper, we developed a new hybrid digital rock construction approach to mimic the pore space of tight sandstone by combining X-ray CT scanning and multiple-point geostatistics algorithm (MPGA). First, a three-dimensional macropore digital rock describing the macroscopic pore structure of tight sandstone was constructed by micro-CT scanning. Then, high-resolution scanning electron microscopy (SEM) was performed on the tight sandstone sample, and the three-dimensional micropore digital rock was reconstructed by MPGA. Finally, the macropore digital rock and the micropore digital rock were superimposed into the full-pore digital rock. In addition, the nuclear magnetic resonance (NMR) response of digital rocks is simulated using a random walk method, and seepage simulation was performed by the lattice Boltzmann method (LBM). The results show that the full-pore digital rock has the same anisotropy and good connectivity as the actual rock. The porosity, NMR response, and permeability are in good agreement with the experimental values.

\section{Introduction}

Looking around the world, with the depletion of conventional fossil energy, unconventional hydrocarbon resources will be the most realistic resource type in this change [1]. However, the production and development of unconventional hydrocarbon resources are still facing many difficulties. From the point of view of production practice, the unconventional reservoir usually has no commercial production and its development often relies on large-scale hydraulic fracturing, which has the characteristics of high initial production, rapid decline, low recovery, and so on [2-4]. Since the macroscopic phenomena are often the result of microfactors, the rock structure and percolation mechanism of unconventional reservoirs studied at microscale are key means to the aforementioned macroproblems and improve the production capacity eventually. Nevertheless, unconventional reservoir rock is usually characterized by low porosity and permeability, fine pore throat, complex pore structure, and developed fracture network [5-7], the laboratory experiment is difficult to carry out, and the routine rock modeling methods such as capillary model are hard to describe the true and microscopic structure of unconventional rocks.

In face of these challenges, digital rock technology is currently expected to be the key to solve the above puzzles in unconventional reservoirs. Digital rock technology originated in the 1970s and was proposed for comprehensively mimicking the real rock by imaging and mathematical algorithm, which has gradually developed into a promising tool in the study of porous media [8-13]. Based on the digital rock models, the microstructure of the rock can be quantitatively characterized and microseepage simulation can be carried out as well. Digital rocks have enabled important 
breakthroughs in understanding how fluid enters pore spaces and the mechanisms of water/oil and gas seepage [14-16].

At present, the methods of digital rock reconstruction mainly include physical experiments, numerical reconstructions, and hybrid modeling. There is an extensive literature on these various approaches, and we summarize here their general philosophy and some of their limitations. Physical experiment methods have high accuracy and are widely used but are vulnerable to the tradeoff between resolution and dimension $[17,18]$. Compared with the physical experiment method, the numerical reconstruction method has the advantages of low cost and high efficiency and can reconstruct different types of digital rocks. However, the drawback of the numerical reconstruction is that there are some differences between the model and the real rock [19-23]. To address the shortcomings of the single modeling method, some scholars have proposed the idea of mixing different modeling methods with each other, i.e., hybrid modeling methods [24-28]. For example, Talukdar et al. combined the truncated Gaussian random field method (TGRFM) with the characteristic of fast modeling speed and the simulated annealing method (SAM) with the characteristic of more accurate modeling and used the images generated by TGRFM as input to SAM to establish a digital rock with higher model quality. Politis et al. and Liu et al. reconstructed $3 \mathrm{D}$ digital rocks by combining the process-based method (PBM) and SAM. The basic process of their modeling is similar. First, use the PBM to construct the initial 3D digital rock and then use the initial model as the input of SAM. When the conditions of SAM are reached, a hybrid digital rock is generated. Compared with the traditional SAM, their method is more efficient in modeling and the model is more accurate. More recently, Lin et al. introduced a hybrid modeling method that combines physical experiments and numerical reconstruction. First, a 3D macropore digital rock was constructed by micro-CT; second, using high-resolution 2D SEM images, a micropore digital rock was constructed by SAM; last, a superposition method was used to construct the digital rock including the full range of pore structures. This method not only keeps the accuracy of the macropore image but also contains microstructure information. Hybrid modeling methods have improved modeling accuracy and efficiency and overcome some limitations of single modeling methods.

In this work, based on the hybrid method combining physical experiments and numerical reconstructions, we further develop a new combination method that integrated macropores constructed by X-ray $\mathrm{CT}$ and micropores reconstructed by MPGA. We utilized micro-CT to image the macropore structure of tight sandstone and constructed the macropore digital rock and used the SEM to image the micropores of tight sandstone and reconstructed the micropore digital rock using MPGA. Through the superposition of micropore and macropore digital rocks, the full-pore digital rock of tight sandstone was obtained, and the physical properties and seepage characteristics of the digital rocks were compared and analyzed.

\section{Sandstone Modeling}

2.1. 3D Macropore Reconstruction by X-Ray CT. Computerized tomography (CT) imaging has the advantages of high accuracy and being nondestructive and is widely used in the field of rock physics $[29,30]$. As illustrated in Figure 1, the working principle of CT is that when X-rays are irradiated and penetrated through the sample, the energy will be attenuated differently and the attenuation process accords with the attenuation formula as follows:

$$
I=I_{0} e^{-\sum_{i} \mu_{i} x_{i}} .
$$

In formula (1), $I$ is the intensity after attenuation, $I_{0}$ is the original intensity, $\mu_{i}$ is the attenuation coefficient of component $i$ to ray, and $x_{i}$ is the length of ray passing through component $i$. When $\mathrm{X}$-rays penetrate the sample, the detector detects the attenuated ray intensity and imitates it on the detector.

The core of CT imaging is to reconstruct the gray image of the sample. There are two basic reconstruction algorithms: analytical method and iterative method. At present, FDK reconstruction algorithm is more commonly used, and the formula of the FDK algorithm is as follows [29, 31-33]:

$$
\begin{aligned}
p^{\prime}(\beta, a, b) & =\left(\frac{R}{\sqrt{R R+a a+b b}} p(\beta, a, b) g^{n}(a)\right), \\
F_{F D K}(x, y, z) & =\int_{0}^{2 \pi} \frac{R^{2}}{U(x, y, \beta)^{2}} p^{\prime}(\beta, a, b) d_{\beta}, \\
a & =R \tan \gamma, \\
b & =\frac{q}{\cos \gamma}, \\
k & =\arctan \frac{q}{R}=\arctan \frac{b}{\sqrt{R R+a a}}, \\
U(x, y, \beta) & =R+x \cos \beta+y \sin \beta .
\end{aligned}
$$

In formula (2), $U(x, y, \beta)$ is the total attenuation coefficient, $q$ is the length of the projection data in a certain direction, $p(\beta, a, b)$ is the collected projection data, $p^{\prime}(\beta, a, b)$ is the weighted filtering of the projection data, $F_{F D K}(x, y, z)$ is the back projection reconstruction of the weighted filter projection data, $g^{n}(a)$ is a one-dimensional filter, $R$ is the orbital radius, $\gamma$ is the fan angle of the cone beam, $\beta$ is the cone angle of the cone beam, and $a$ and $b$ are the positions on the detector.

We used an UltraXRM-L200 CT scanner to image the macropores $(>1.2 \mu \mathrm{m})$ of the tight sandstone. The sandstone sample was selected and made into a cylinder with approximately $1.2 \mathrm{~mm}$ diameter and $1.4 \mathrm{~mm}$ length, and its porosity was $12.16 \%$ and gas permeability was $0.413 \times 10^{-3} \mu \mathrm{m}^{2}$. The resolution of CT imaging was 1.2 micron/pixel, and $996 \mathrm{CT}$ grayscale images were obtained. The original grayscale images (Figure 2) were preprocessed through contrast enhancement and a median filter. We used an improved threshold segmentation method considering 


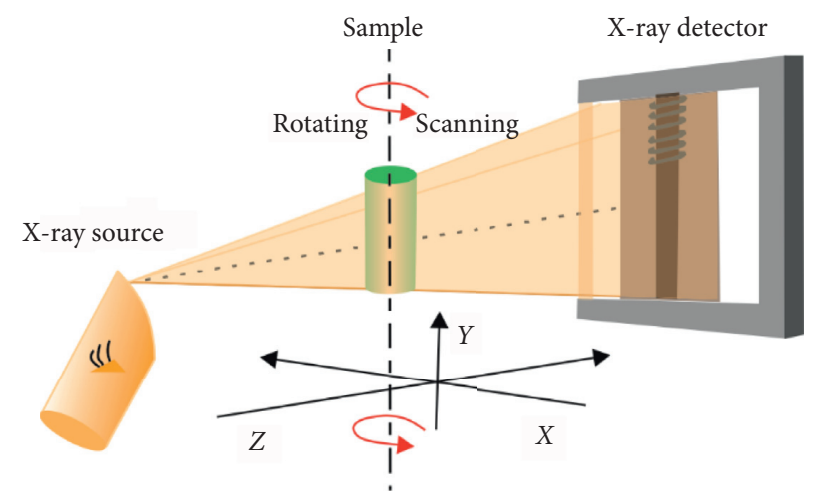

Figure 1: Schematic diagram of X-ray CT imaging [28].

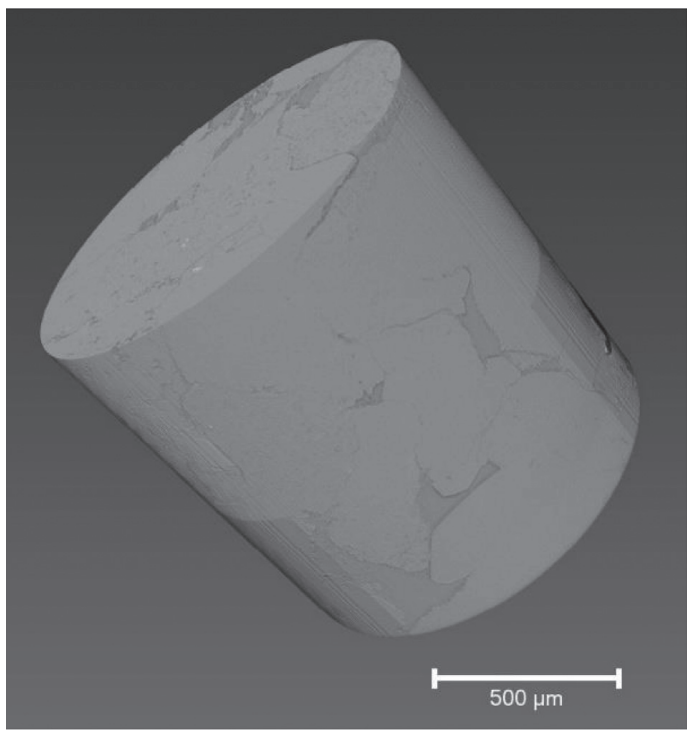

(a)

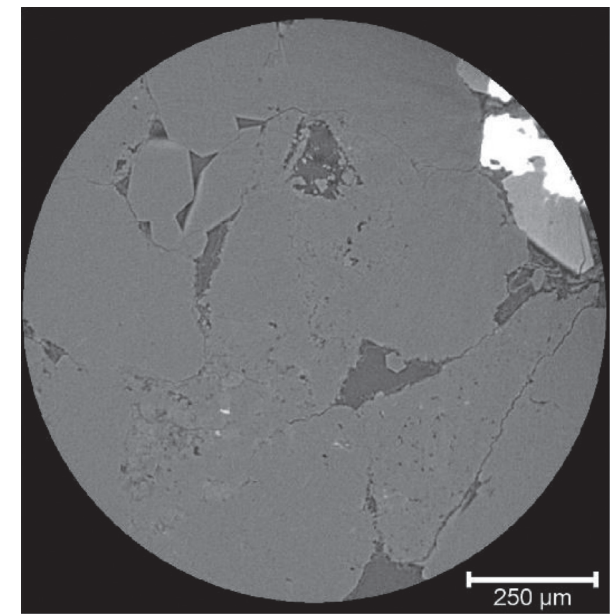

(b)

Figure 2: 3D grayscale image of the tight sandstone (a) and 2D grayscale image cross section (b).

pore fractal characteristics to make the optimal segmentation [34]. Figure 3 shows a binary image of tight sandstone by micro-CT (white is pore; black is solid). Figure 4 presents a $3 \mathrm{D}$ macropore digital rock obtained by processing $\mathrm{CT}$ images, whose physical size is $0.12 \mathrm{~mm} \times 0.12 \mathrm{~mm} \times 0.12 \mathrm{~mm}$ and voxel size is $100 \times 100 \times 100$. From CT scanning, we can see that the sample contains primary pores and secondary pores. The primary pores have straight edges, clean pore interiors, and large pore radii. The secondary pores mainly include intragranular pores and marginal pores of feldspar and detritus, a small amount of dissolved pores in carbonate cements, and the intercrystalline pores in clay minerals.

2.2. 3D Micropore Reconstruction by MPGA. To characterize smaller pores, we imaged the same sample scanned by CT using scanning electron microscopy (SEM); the imaging resolution of SEM images is 0.3 micron/pixel (Figure 5(a)); the same image processing used for the CT images was used to generate the binary SEM images, as shown in Figure 5(b).
Multiple-point geostatistics algorithm (MPGA) was originally used in geostatistics to deal with continuous geological entities at reservoir scale. The basis of MPGA is to replace the variation function of two-point geological statistics algorithm with a training image. The basic idea of this algorithm is to extract the image patterns of features from the training images and then restore these patterns to the final model $[20,21,35]$. In this work, we used MPGA to produce micropores in sandstone, and the basic steps are as follows:

(1) Using the two-dimensional SEM binary image as the initial training image, the training image is scanned by the determined two-dimensional data template to construct a search tree.

(2) Extract $0.5 \%$ of the pixel points from the two-dimensional training image used for each simulation as the horizontal direction (such as the $x-y$ direction) condition data and assign it to the nearest network node. A two-dimensional slice such as the $y-z$ 


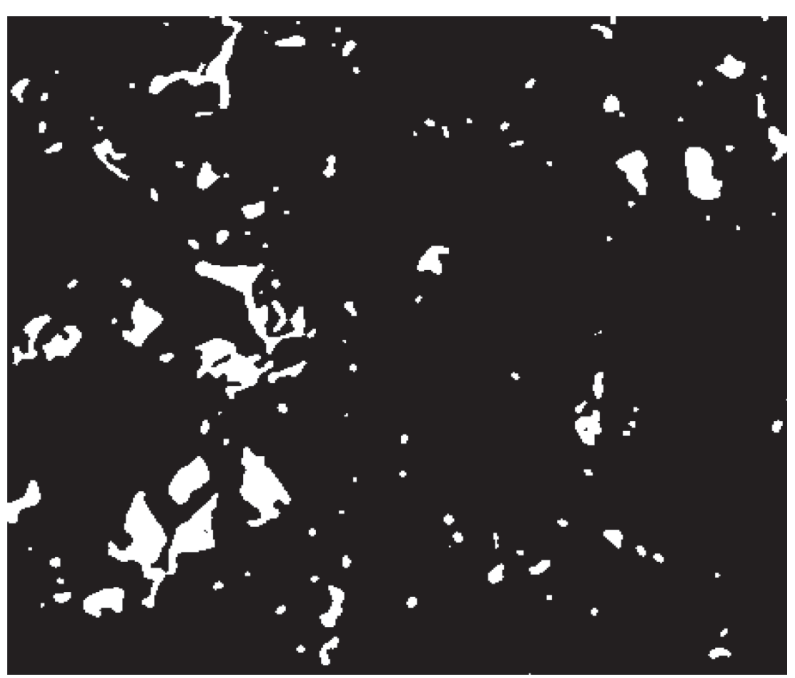

FIGURE 3: Binary image of tight sandstone by micro-CT (white is pore; black is solid).

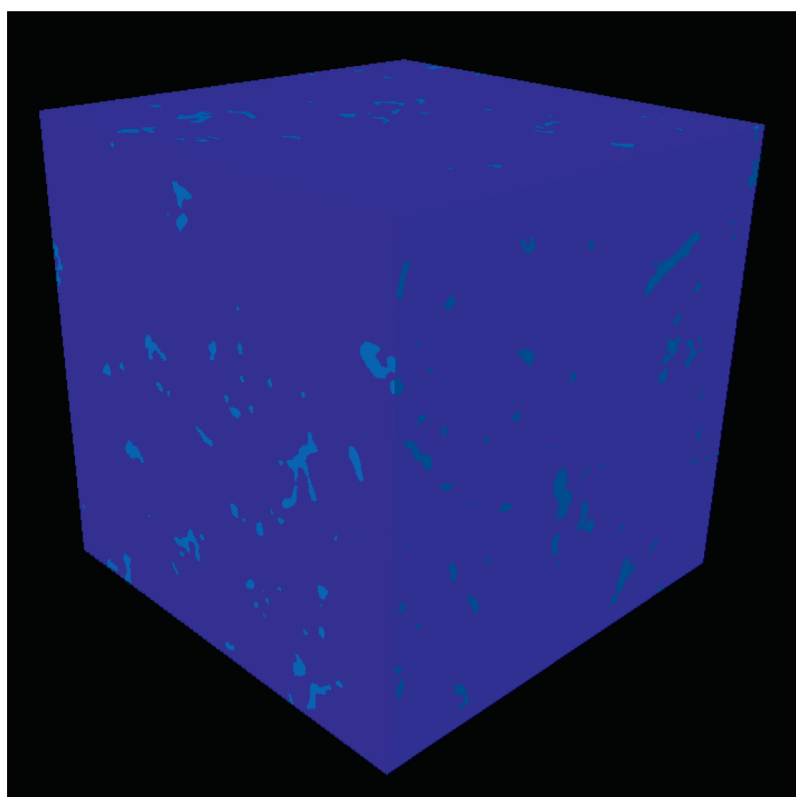

Figure 4: 3D macropore digital rock using X-ray CT scanning method (blue is solid; green is pore; black is background).

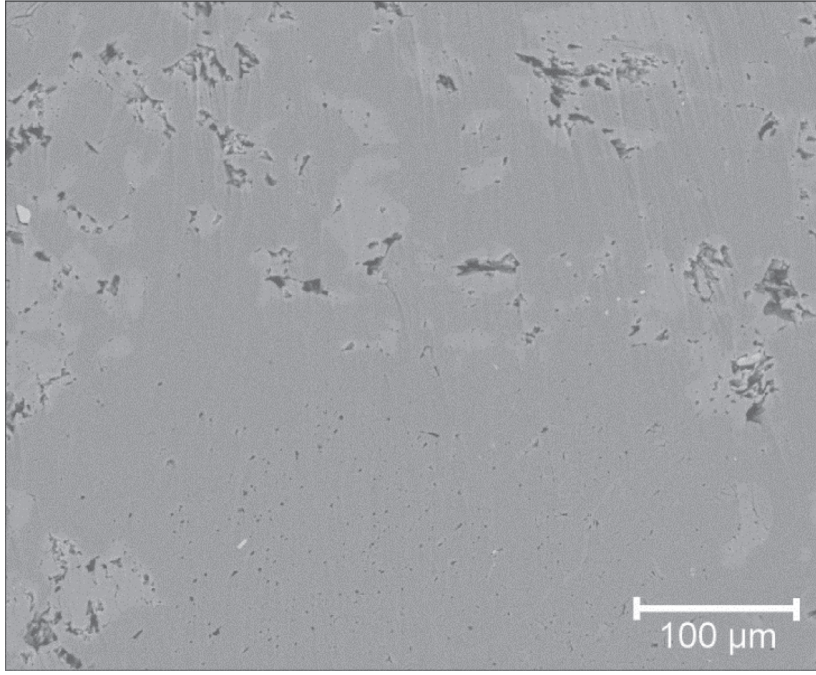

(a)

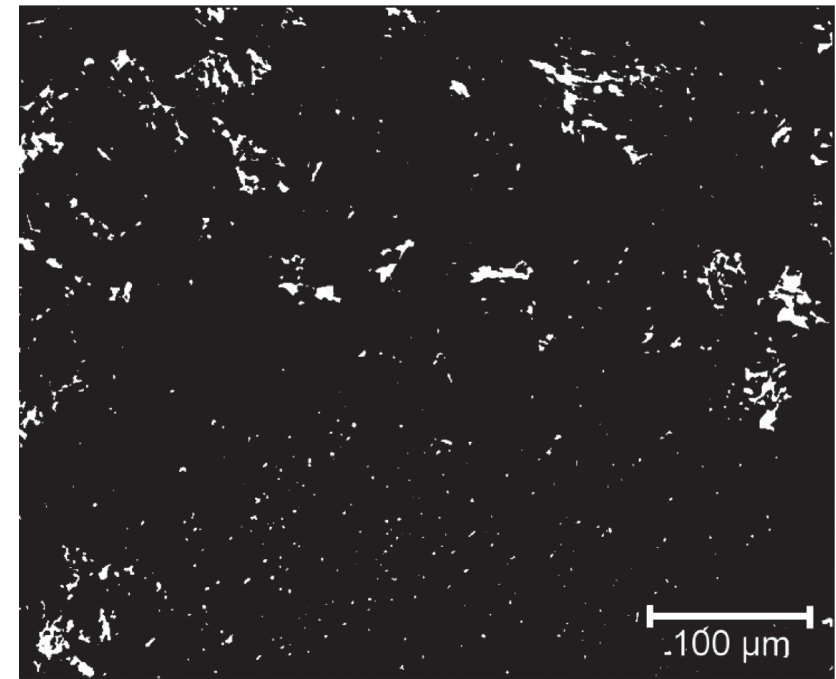

(b)

FIGURE 5: Grayscale image (a) and binary image (b) of tight sandstone by SEM (white is pore; black is solid). 
direction or the $x-z$ direction is selected as a vertical direction condition for simulation.

(3) Sequentially access all the nodes to be simulated with the determined random path, and for each node on the path, the condition data event is extracted together with the data template of the same size in step (1) and the condition data obtained in step (2). The conditional probability distribution function is obtained from the search tree, and the Monte Carlo method is used to extract the simulated value of the point; the simulation is continued as a new condition of the subsequent simulation until the two-dimensional image is generated.

(4) The two-dimensional image simulated in step (3) is used as the training image to continue the simulation in the next step, and the first three steps are repeated until the next layer of the image is generated.

(5) Repeat the first 4 steps until the simulation yields $N$ new two-dimensional images.

In the process of simulation, the edge processing of the reconstructed image uses the target edge probability function. Since the simulated digital rock has only two parts of the pore and the skeleton, the porosity of the two-dimensional SEM binary is used as its target edge value and the servosystem is used to ensure the accuracy of the simulation results. The simulated $N$ images are sequentially superimposed to obtain a three-dimensional digital rock of $N \times N \times N$. For the processing of excessive condition data, the method of setting the lower limit value is adopted. If the number of repetitions obtained by scanning the training image using the template is lower than the lower limit value, the condition data farthest from the center point is removed, the data are rescanned until the set lower limit value is reached, and then the conditional probability value of the point is obtained at this time. For the processing of only one conditional data, the edge probability at this time can be used as the conditional probability.

Figure 6 shows the micropore digital rock constructed by the MPGA, whose physical size is $0.12 \mathrm{~mm} \times 0.12 \mathrm{~mm} \times$ $0.12 \mathrm{~mm}$ and the voxel size is $400 \times 400 \times 400$.

2.3. Full-Pore Model. We used the superposition method [27] to integrate two digital rocks. According to the resolution ratio of the macropore digital rock to micropore digital rock $i(i=4)$, the voxels of macropore digital rock were divided into $i \times i \times i$ voxels. The voxel refinement diagram is shown in Figure 7.

The pores of both digital rocks are superimposed, and the superimposed pores $I_{S}$ is

$$
I_{S}=I_{A} U I_{B}
$$

where $I_{A}$ and $I_{B}$ represent the pore space of macropore digital rock and micropore digital rock, respectively. Because the pore pixels and solid pixels of the binary image are characterized by 0 and 1, respectively, the superposition operation is

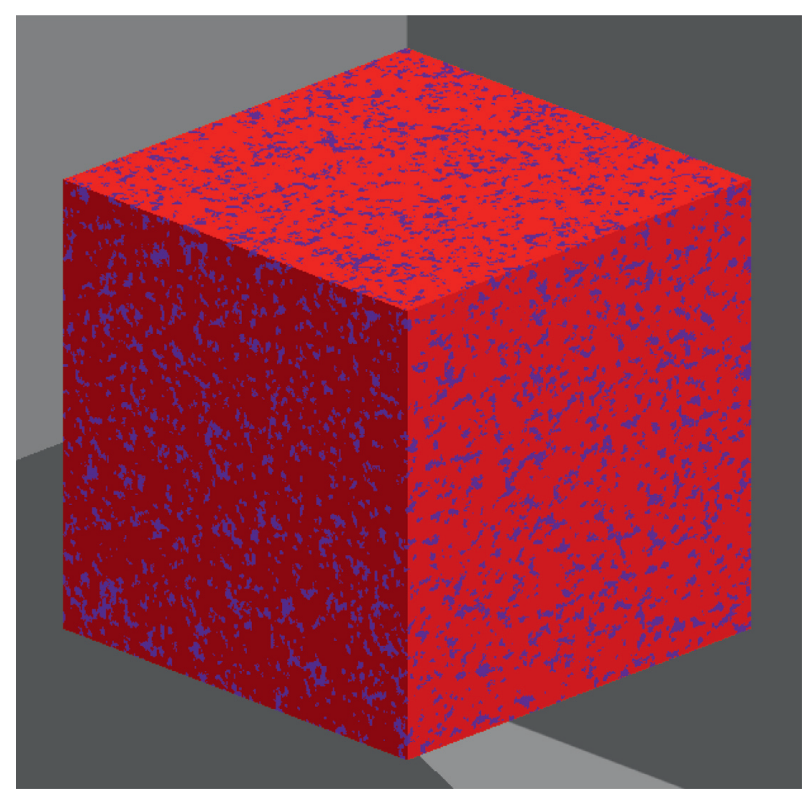

FIGURE 6: 3D micropore digital rock by MPGA (blue is pore; red is solid).

$$
\begin{aligned}
& 0+0=0 \\
& 0+1=0 \\
& 1+0=0 \\
& 1+1=1
\end{aligned}
$$

Figure 8 presents the full-pore digital rock of tight sandstone constructed by the superposition method, whose voxel size is $400 \times 400 \times 400$ and resolution is 0.3 micron/ pixel.

\section{Structural Characteristics of the Sandstone Model}

3.1. Connectivity Analysis. We calculate the porosity of the digital rocks by using the following equation:

$$
\phi=\frac{V_{p}}{V_{p}+V_{s}},
$$

where $V_{p}$ is the volume of all pores, which contains the connected pores and the dead pores in the digital rock, $\mathrm{m}^{3}$. $V_{s}$ is the volume solid matrix, $\mathrm{m}^{3}$. Here, the pore volume and solid matrix volume of digital rocks are calculated by counting the number of voxels labeled as 0 and the number of voxels labeled as 1 , respectively.

We evaluated the connectivity of digital rocks using the connected porosity. Connected porosity $\phi_{c}$ is defined by the following formula:

$$
\phi_{c}=\frac{V^{\prime}}{V_{p}+V_{s}},
$$

where $V^{\prime}$ is the volume of connected pores, $\mathrm{m}^{3}$.

The calculation results are shown in Table 1 . The results show that the porosity of the full-pore digital rock is slightly 


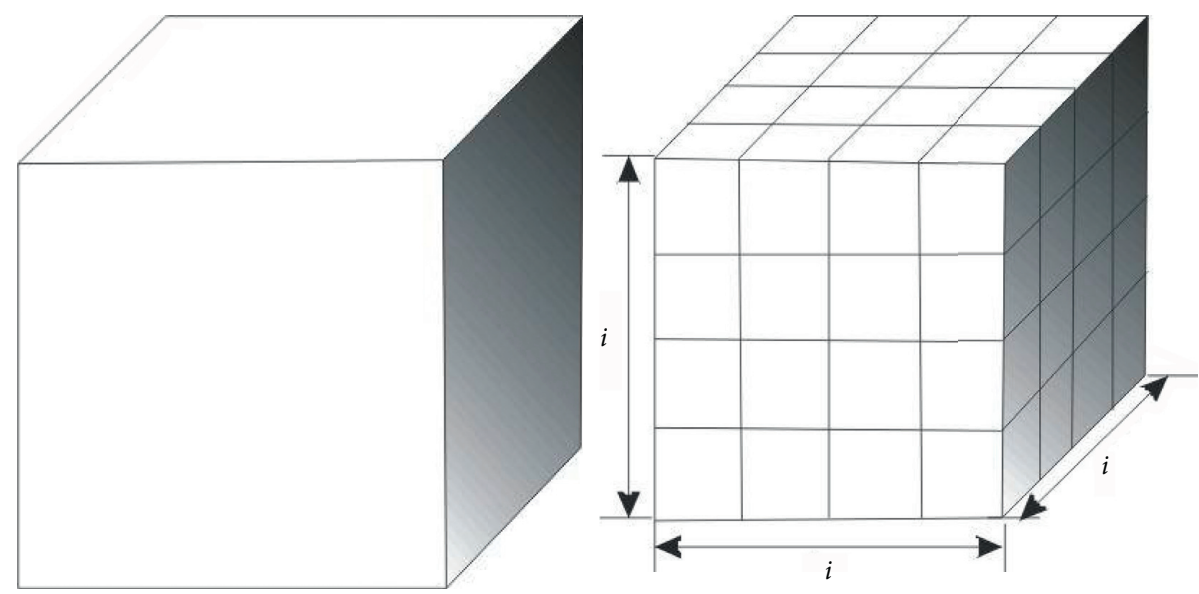

Figure 7: Schematic diagram of voxel refinement.

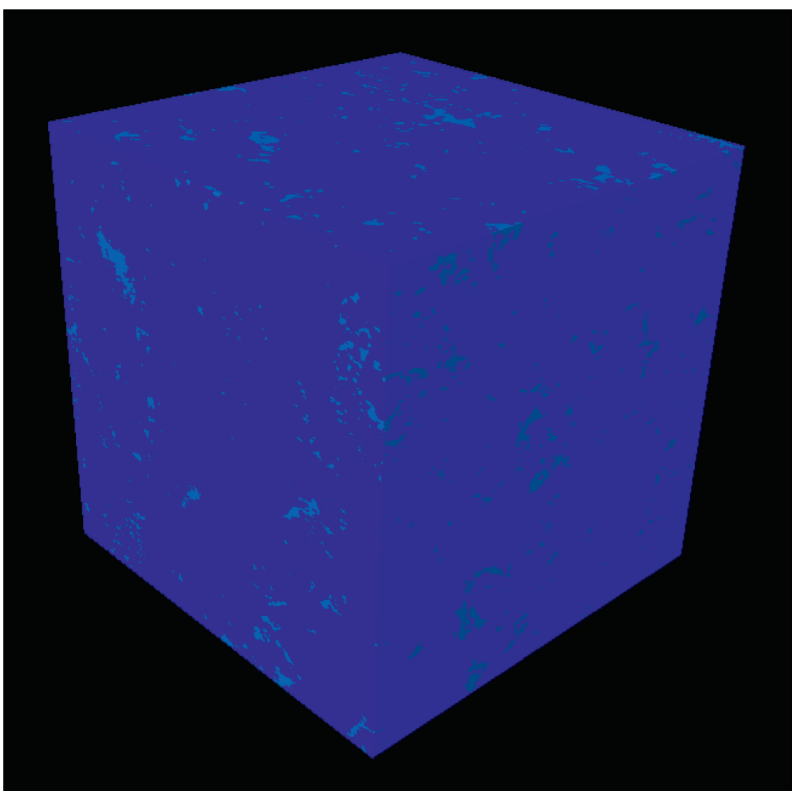

Figure 8: Full-pore digital rock of tight sandstone by the new method (blue is solid; green is void; black is background).

TABLE 1: Porosity comparison between digital rocks and real sandstone.

\begin{tabular}{lcccc}
\hline Parameters & Macropore digital rock & Micropore digital rock & Full-pore digital rock & Real rock \\
\hline Porosity (\%) & 11.23 & 11.76 & 13.83 & - \\
Connected porosity (\%) & 9.19 & 10.47 & 11.81 & 12.16 \\
\hline
\end{tabular}

higher than that of the macropore digital rock and the micropore digital rock. The connected porosity of the fullpore digital rock is significantly higher than that of the other two digital rocks, which proves that the addition of micropores improves the pore connectivity of digital rock. In addition, the porosity of the full-pore digital rock is higher than the measured porosity of the actual rock, and the connected porosity is close to the measured porosity, which is consistent with the fact that the laboratory uses nitrogen to measure the rock porosity and the isolated pore cannot be measured. The measured porosity is actually equal to the connected porosity. Due to the strong heterogeneity of the tight sandstone pore structure, a single simulation method cannot capture the total pore volume, while the full-pore digital rock has better pore connectivity and better consistency with the actual rock in terms of connected porosity.

3.2. NMR Response. In this section, a random walk method is used to simulate the nuclear magnetic resonance (NMR) [36]. This involves simulating the Brownian motion of a diffuse particle called a walker. These walkers diffuse in the pore space, and when they come in contact with a solid surface, their chances of being killed are limited. The 
life-time distribution of the walkers is a decay in magnetization, which can be inverted to a $\mathrm{T} 2$ distribution by using a curvature-smoothing regularization method [37].

It is acknowledged that the short T2 time in the T2 spectrum represents the small pore space and the long T2 time represents the big pore space [38-40]. We compared the results of the laboratory NMR test on the sandstone sample with the simulation NMR results on digital rocks. The laboratory NMR test was carried out on the tight sandstone core saturated with water using the low-field NMR detection device (MacroMR12-150H-I, Niumag, China), and the NMR test mainly refers to the core analysis method (GBT 29172-2012, China) and the laboratory measurement specification of NMR parameters of rock samples (SY-T6490-2014, China). From Figure 9, we can see that tight sandstone has typical dual pore characteristics, showing the bimodal distribution on the NMR T2 spectrum. Simulation results of the digital rocks show that the T2 distributions of the macropore and micropore digital rocks are narrower and have single modal distributions, the T2 value of micropore digital rock is mainly distributed in the short T2 time range, and the T2 value of macropore digital rock is dominant in the long T2 time, implying that the macropore digital rock mainly mimics the relatively large pores, while the micropore digital rock mainly mimics the relatively small pores. For the full-pore digital rock, the simulated T2 distribution has a bimodal distribution and shows good agreement with the experimental result, although there is a small difference as is attributed to the loss of some fine details of the pore structure $(<0.3$ microns $)$ in tight sandstone.

By comparative analysis, it can be seen that single resolution imaging and single modeling method cannot reconstruct the pore structure and restore connectivity of tight sandstone digital rock. Combined with X-ray CT and SEM, we can capture micropores and macropores in a complete model using the hybrid modeling method. On the whole, the full-pore digital rock shows good performance in the multiscale pore structure simulation of tight sandstone. At the same time, in the study of tight reservoir, we should also pay special attention to the influence of submicron pores on rock seepage ability.

\section{Seepage Properties}

We carried out the permeability evaluation on digital rocks by using the lattice Boltzmann method (LBM) [27, 41, 42]. The LBM utilizes the collision, and migration of virtual particle swarms with different velocity directions on discrete spatial grid points to characterize fluid flow, which can effectively simulate complex flows such as porous media flow, convection current, and multiphase flow. LBM is a mesoscopic method, and the basic idea of this method is based on the theory of molecular dynamics. There are three commonly used 3D lattice Boltzmann models, which are divided according to the number of discrete velocity components in $3 \mathrm{D}$ space in the direction of fluid continuous motion. Generally, the common dispersion is 15,19 , and 24 components, corresponding to D3Q15, D3Q19, and D3Q24 models, respectively. The more discrete velocity components, the higher the accuracy of the numerical simulation, but at the same time, it brings the calculation problems such as large amount of data and slow simulation speed. Here, we adopt the D3Q19 3D lattice Boltzmann model, and Figure 10 shows the lattice structure, and the velocity discrete Boltzmann-BGK equation is as follows:

$$
f_{i}(r, t)-f_{i}\left(r+e_{i} \delta_{t}, t+\delta_{t}\right)=\frac{1}{\tau}\left[f_{i}(r, t)-f_{\text {eqi }}(r, t)\right],
$$

where, $f_{i}(r, t)$ is the distribution function of the particle in the $i$ direction at $t$ time and position $r(x, y, z), e_{i}$ is the velocity in the $i$ direction, $\tau$ is the relaxation time, and $\delta_{t}$ is the time step, $f_{\text {eqi }}(r, t)$ is the equilibrium distribution function.

The discrete velocity direction is as follows:

$$
e_{i}= \begin{cases}(0,0,0), & i=0 \\ ( \pm 1,0,0),(0, \pm 1,0),(0,0, \pm 1), & i=1, \ldots, 6 \\ ( \pm 1, \pm 1,0),( \pm 1,0, \pm 1),(0, \pm 1, \pm 1), & i=7, \ldots, 18\end{cases}
$$

Equilibrium distribution function is as follows:

$$
f_{\text {eqi }}(r, t)=\rho \omega_{i}\left[1+\frac{e_{i} u}{c_{s}^{2}}+\frac{\left(e_{i} u\right)^{2}}{2 c_{s}^{4}}-\frac{u^{3}}{2 c_{s}^{2}}\right],
$$

where $c_{s}$ is the lattice sound velocity, dimensionless with $c_{s}=$ $1 / \sqrt{3}$ and $\omega_{i}$ is the weight factor, $\omega_{i}=1 / 3, \quad i=0, \omega_{i}=$ $1 / 18, \quad i=1, \ldots, 6, \omega_{i}=1 / 36, \quad i=7, \ldots, 18$.

Local macrolattice density $\rho(r, t)$, lattice velocity $u(r, t)$, lattice pressure $p(r, t)$, and lattice viscosity $\mu(r, t)$ can be represented by

$$
\begin{aligned}
& \rho(r, t)=\sum_{i} f_{i}(r, t), \\
& p(r, t)=c_{s}^{2} \rho(r, t), \\
& u(r, t)=\frac{\sum_{i} f_{i}(r, t) e_{i}}{\rho(r, t)}, \\
& \mu(r, t)=\frac{2 \tau-1}{6} .
\end{aligned}
$$

The inlet and outlet boundary is pressure boundary, the initial lattice velocity is 0 , and the initial lattice density is 1 .

We used the LBM to simulate the seepage process of macropore digital rock (Figure 4), micropore digital rock (Figure 6), and full-pore digital rock (Figure 8) and obtained the inherent permeability of digital rocks. The inherent permeability of full-pore digital rock is $0.377 \times 10^{-3} \mu \mathrm{m}^{2}$, the inherent permeability of micropore digital rock is $0.093 \times 10^{-3} \mu \mathrm{m}^{2}$, and the inherent permeability of macropore digital rock is $0.216 \times 10^{-3} \mu \mathrm{m}^{2}$. The simulation results show that the permeability of full-pore digital rock is larger than that of micropore digital rock and macropore digital rock, suggesting that micropores can greatly improve the conductivity of digital rocks. In addition, the calculated 


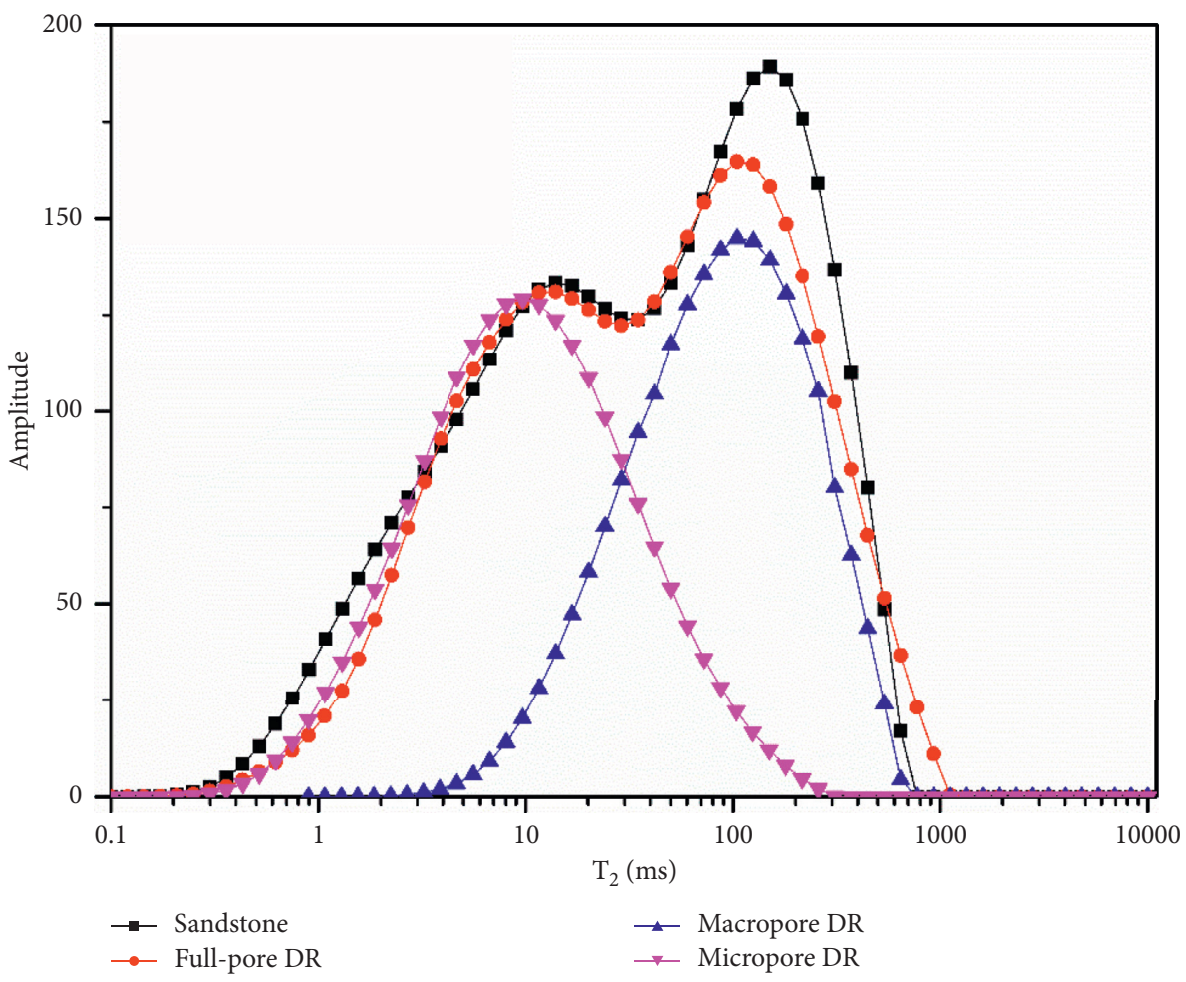

FIGURE 9: Comparison of NMR response between the simulated results of digital rocks and the experimental result of real sandstone.

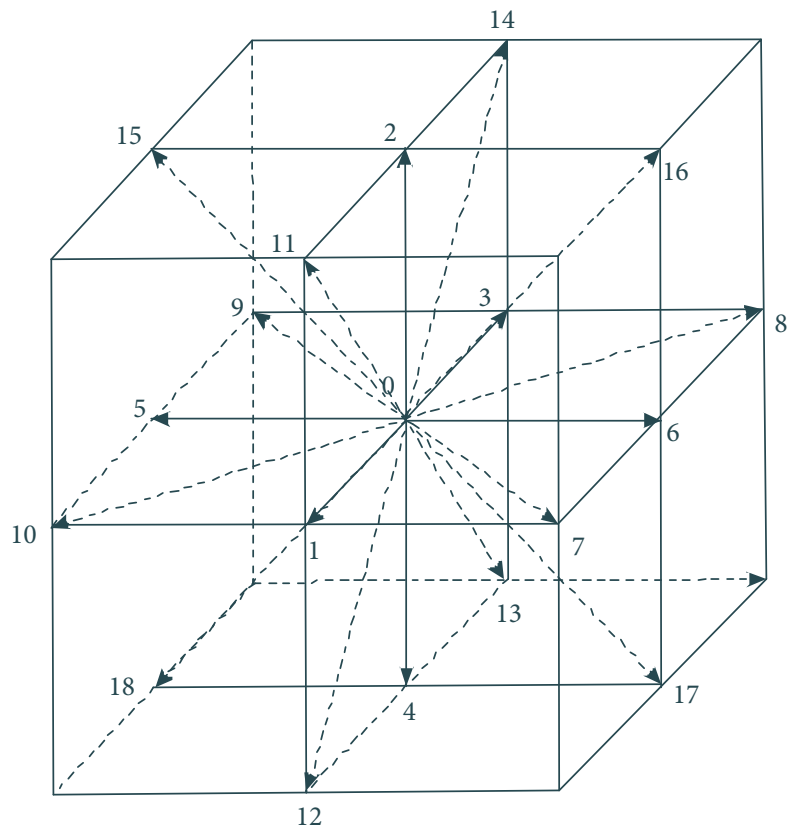

FIGURE 10: D3Q19 lattice structure model.

permeability of the full-pore digital rock is consistent with the experimental result $\left(0.413 \times 10^{-3} \mu \mathrm{m}^{2}\right)$, which shows that the permeability of the full-pore digital rock is similar to that of the real tight sandstone. The permeability ratio of fullpore digital rock to macropore digital rock is 1.75 , which indicates that micropores have great influence on the permeability of digital rock model. Furthermore, we should fully consider and study the transport characteristics of submicron pores in tight sandstone.

\section{Conclusions}

In this study, we proposed a new strategy by combining the $\mathrm{X}$-ray CT scanning method and multiple-point geostatistics 
algorithm (MPGA) in digital rock. Unlike the existing numerical reconstruction methods, this method is a hybrid of physical experiments and numerical reconstruction, so it not only has the accuracy of the physical experiment method but also has the convenience of the numerical reconstruction method. We compared and analyzed the pore structure and seepage characteristics of the full-pore digital rock constructed by the new hybrid method, the macropore digital rock constructed by the X-ray CT scanning method, and the micropore digital rock constructed by MPGA. The results show that the new hybrid method can better simulate the real rock, and the pore structure and seepage properties of the full-pore digital rock are basically consistent with those of the actual rock. In addition, this study also shows that single-resolution imaging and the single modeling method cannot produce the satisfactory digital rock model, especially for tight sandstone with a multiscale pore system. Furthermore, tight sandstones are generally rich in clay minerals (such as kaolinite, illite, and chlorite), which has great effect on macroelectrical conductivity, elastic modulus, and permeability, and it is thus an interesting direction to construct multiphase multicomponent digital core in the future.

\section{Data Availability}

The data used to support the findings of this study are available from the corresponding author upon request.

\section{Conflicts of Interest}

The authors declare that they have no conflicts of interest regarding the publication of this work.

\section{Acknowledgments}

This work was financially supported by the National Science and Technology Major Project (Grant no. 2016ZX05067004003). The author thanks Huang Lei and Chengwu Li very much for helping with the experiments and paper writing.

\section{References}

[1] C. Zou, G. Zhai, G. Zhang et al., "Formation, distribution, potential and prediction of global conventional and unconventional hydrocarbon resources," Petroleum Exploration and Development, vol. 42, no. 1, pp. 14-28, 2015.

[2] Z. Y. Wang, Z. M. Yang, Y. H. Ding, W. Lin, Y. He, and $X$. Duan, "A generalized capillary imbibition model for porous media in tight reservoirs," Advances in Civil Engineering, vol. 2018, Article ID 4148734, 8 pages, 2018.

[3] J. Du, H. Liu, D. Ma, J. Fu, Y. Wang, and T. Zhou, "Discussion on effective development techniques for continental tight oil in China," Petroleum Exploration and Development, vol. 41, no. 2, pp. 217-224, 2014.

[4] H. Zhang, Z. Shi, G. Wang, X. Sun, et al.,C. Liu, "Large earthquake reshapes the groundwater flow system: insight from the water-level response to earth tides and atmospheric pressure in a deep well," Water Resources Research, vol. 55, no. 5, pp. 4207-4219, 2019.
[5] X. Zhao, Z. Yang, W. Lin, S. Xiong, and Y. Wei, "Characteristics of microscopic pore-throat structure of tight oil reservoirs in Sichuan Basin measured by rate-controlled mercury injection," Open Physics, vol. 16, no. 1, pp. 675-684, 2018.

[6] X. L. Zhao, Z. M. Yang, W. Lin et al., "Fractal study on pore structure of tight sandstone based on full-scale map," International Journal of Oil, Gas and Coal Technology, vol. 1, no. 1, 2019.

[7] K. Xie, X. Lu, Q. Li, W. Jiang, and Q. Yu, “Analysis of reservoir applicability of hydrophobically associating polymer," SPE Journal, vol. 21, no. 1, pp. 001-009, 2016.

[8] M. Joshi, "A class of stochastic models for porous media," Ph.D. thesis, University of Kansas, Lawrence, Kansas, 1974.

[9] J. A. Quiblier, "A new three-dimensional modeling technique for studying porous media," Journal of Colloid and Interface Science, vol. 98, no. 1, pp. 84-102, 1984.

[10] S. Bryant and M. Blunt, "Prediction of relative permeability in simple porous media," Physical Review A, vol. 46, no. 4, pp. 2004-2011, 1992.

[11] R. D. Hazlett, "Statistical characterization and stochastic modeling of pore networks in relation to fluid flow," Mathematical Geology, vol. 29, no. 6, pp. 801-822, 1997.

[12] C. L. Y. Yeong and S. Torquato, "Reconstructing random media. II. Three-dimensional media from two-dimensional cuts," Physical Review E, vol. 58, no. 1, pp. 224-233, 1998.

[13] P. E. Øren and S. Bakke, "Reconstruction of Berea sandstone and pore-scale modelling of wettability effects," Journal of Petroleum Science and Engineering, vol. 39, no. 3-4, pp. 177-199, 2003.

[14] K. Singh, M. Jung, M. Brinkmann, and R. Seemann, "Capillary-Dominated fluid displacement in porous media," $A n$ nual Review of Fluid Mechanics, vol. 51, no. 1, pp. 429-449, 2019.

[15] J. Yao, H. Sun, A. Li et al., "Modern system of multiphase flow in porous media and its development trend," Chinese Science Bulletin, vol. 63, no. 4, pp. 425-451, 2018.

[16] Z. M. Yang, X. L. Zhao, S. C. Xiong et al., "Research progress on microstructure characterization of pore throat for tight oil reservoirs," Science \& Technology Review, vol. 37, no. 5, pp. 89-98, 2019.

[17] H. Izadi, M. Baniassadi, A. Hasanabadi et al., "Application of full set of two point correlation functions from a pair of $2 \mathrm{D}$ cut sections for 3D porous media reconstruction," Journal of Petroleum Science and Engineering, vol. 149, pp. 789-800, 2017.

[18] L. Tomutsa, D. B. Silin, and V. Radmilovic, "Analysis of chalk petrophysical properties by means of submicron-scale pore imaging and modeling," SPE Reservoir Evaluation \& Engineering, vol. 10, no. 3, pp. 285-293, 2007.

[19] R. D. Hazlett, "Simulation of capillary-dominated displacements in microtomographic images of reservoir rocks," Multiphase Flow in Porous Media, vol. 20, no. 1-2, pp. 21-35, 1995.

[20] H. Okabe and M. J. Blunt, "Prediction of permeability for porous media reconstructed using multiple-point statistics," Physical Review E, vol. 70, no. 6, Article ID 066135, 2004.

[21] P. Tahmasebi, A. Hezarkhani, and M. Sahimi, "Multiple-point geostatistical modeling based on the cross-correlation functions," Computational Geosciences, vol. 16, no. 3, pp. 779-797, 2012.

[22] Y. Keehm, T. Mukerji, and A. Nur, "Permeability prediction from thin sections: $3 \mathrm{D}$ reconstruction and Lattice-Boltzmann 
flow simulation," Geophysical Research Letters, vol. 31, no. 4, Article ID L04606, 2004.

[23] P. E. Oren and S. Bakke, "Process based reconstruction of sandstones and prediction of transport properties," Transport in Porous Media, vol. 46, no. 2-3, pp. 311-343, 2002.

[24] M. S. Talukdar and O. Torsaeter, "Reconstruction of chalk pore networks from 2D backscatter electron micrographs using a simulated annealing technique," Journal of Petroleum Science and Engineering, vol. 33, no. 4, pp. 265-282, 2002.

[25] M. G. Politis, E. S. Kikkinides, M. E. Kainourgiakis, and A. K. Stubos, "A hybrid process-based and stochastic reconstruction method of porous media," Microporous and Mesoporous Materials, vol. 110, no. 1, pp. 92-99, 2008.

[26] X. Liu, J. Sun, and H. Wang, "Reconstruction of 3-D digital cores using a hybrid method," Applied Geophysics, vol. 6, no. 2, pp. 105-112, 2009.

[27] W. Lin, X. Li, Z. Yang et al., "Construction of dual pore 3-D digital cores with a hybrid method combined with physical experiment method and numerical reconstruction method," Transport in Porous Media, vol. 120, no. 1, pp. 227-238, 2017.

[28] W. Lin, X. Li, Z. Yang et al., "Multiscale digital porous rock reconstruction using template matching," Water Resources Research, vol. 55, no. 8, pp. 6911-6922, 2019.

[29] M. Zha, X. Y. Yin, L. Jiang et al., "Application of CT technology in petroleum exploration and development," Geological Science and Technology Information, vol. 36, no. 4, pp. 228-235, 2017.

[30] W. Lin, Z. Yang, X. Li et al., "A method to select representative rock samples for digital core modeling," Fractals, vol. 25, no. 4, Article ID 1740013, 2017.

[31] L. A. Feldkamp, L. C. Davis, and J. W. Kress, "Practical conebeam algorithm," Journal of the Optical Society of America A, vol. 1, no. 6, pp. 612-619, 1984.

[32] M. Defrise and R. Clack, "A cone-beam reconstruction algorithm using shift-variant filtering and cone-beam backprojection," IEEE Transactions on Medical Imaging, vol. 13, no. 1, pp. 186-195, 1994.

[33] M. Grass, T. Köhler, and R. Proksa, "3D cone-beam CT reconstruction for circular trajectories," Physics in Medicine and Biology, vol. 45, no. 2, pp. 329-347, 2000.

[34] W. Lin, X. Li, Z. Yang et al., "A new improved threshold segmentation method for scanning images of reservoir rocks considering pore fractal characteristics," Fractals, vol. 26, no. 2, Article ID 1840003, 2018.

[35] L. Zhang, J. M. Sun, Z. Q. Sun et al., "Application of multiplepoint geostatistics in $3 \mathrm{D}$ pore structure model reconstruction," Journal of China University of Petroleum, vol. 36, no. 2, pp. 105-109, 2012.

[36] O. Talabi, S. AlSayari, S. Iglauer, and M. J. Blunt, "Pore-scale simulation of NMR response," Journal of Petroleum Science and Engineering, vol. 67, no. 3-4, pp. 168-178, 2009.

[37] S. Chen, D. Georgi, S. Fang, J. Salyer, and D. Shorey, "Optimization of NMR logging acquisition and processing: SPE 56766," in Proceedings of the SPE 74th Annual Conference and Technical Exhibition (ATCE), Houston, TX, USA, Octobar 1999.

[38] Z. M. Yang, Z. Z. Ma, Y. T. Luo, Y. Zhang, H. Guo, and W. Lin, "A measured method for in situ viscosity of fluid in porous media by nuclear magnetic resonance," Geofluids, vol. 2018, Article ID 9542152, 8 pages, 2018.

[39] X. L. Zhao, Z. M. Yang, W. Lin et al., "Study on pore structures of tight sandstone reservoirs based on nitrogen adsorption, high-pressure mercury intrusion, and rate-controlled mercury intrusion," Journal of Energy Resources Technology-
Transactions of the ASME, vol. 141, no. 11, Article ID 112903, 2019.

[40] Q. Xiao, X. Zhao, W. Lin, X. Huang, and Z. Song, "Application of NMR to test sandstone stress sensitivity of the Dongfang X gas field, China," IEEE Access, vol. 7, pp. 95212-95223, 2019.

[41] M. R. Rezaie and M. Norouzi, "Numerical investigation of MHD flow of non-Newtonian fluid over confined circular cylinder: a lattice Boltzmann approach," Journal of the Brazilian Society of Mechanical Sciences and Engineering, vol. 40, no. 4, p. 185, 2018.

[42] W. Lin, X. Z. Li, Z. M. Yang et al., "Modeling of 3D rock porous media by combining X-ray CT and Markov chain Monte Carlo," Journal of Energy Resources Technology, vol. 142, no. 1, Article ID 013001, 2020. 